\title{
Effects of Mn Substitution on Crystallographic and Magnetic Properties of Li-Zn-Cu Ferrites
}

\author{
Young Bae Lee ${ }^{2}$, Won-Ok Choi ${ }^{1}$, and Kwang Pyo Chae ${ }^{1 *}$ \\ ${ }^{1}$ Department of Nano Science and Mechanical Engineering, \\ ${ }^{2}$ Nanotechnology Research Center, Konkuk University, Chungju 380-701, Korea
}

(Received 16 March 2014, Received in final form 25 May 2014, Accepted 23 June 2014)

\begin{abstract}
The effects of manganese substitution on the crystallographic and magnetic properties of $\mathrm{Li}-\mathrm{Zn}-\mathrm{Cu}$ ferrite, $\mathrm{Li}_{0.5} \mathrm{Zn}_{0.2} \mathrm{Cu}_{0.4} \mathrm{Mn}_{\mathrm{x}} \mathrm{Fe}_{2.1-\mathrm{x}} \mathrm{O}_{4}(0.0 \leq \mathrm{x} \leq 0.8)$, were investigated. Ferrites were synthesized via a conventional ceramic method. We confirmed the formation of crystallized particles using X-ray diffraction, field emission scanning electron microscopy and Mössbauer spectroscopy. All of the samples showed a single phase with a spinel structure, and the lattice constants linearly decreased as the substituted manganese content increased, and the particle size of the samples also somewhat decreased as the doped manganese content increased. All the Mössbauer spectra can be fitted with two Zeeman sextets, which are the typical spinel ferrite spectra of $\mathrm{Fe}^{3+}$ with A- and B-sites, and one doublet. The cation distribution was determined from the variation of the Mössbauer parameters and of the absorption area ratio. The magnetic behavior of the samples showed that an increase in manganese content led to a decrease in the saturation magnetization, whereas the coercivity was nearly constant throughout. The maximum saturation magnetization was $73.35 \mathrm{emu} / \mathrm{g}$ at $\mathrm{x}=0.0$ in $\mathrm{Li}_{0.5} \mathrm{Zn}_{0.2} \mathrm{Cu}_{0.4} \mathrm{Mn}_{\mathrm{x}} \mathrm{Fe}_{2.1-\mathrm{x}} \mathrm{O}_{4}$.
\end{abstract}

Keywords : $\mathrm{Li}-\mathrm{Zn}-\mathrm{Cu}$ ferrite, Mössbauer spectroscopy, cation distribution, saturation magnetization, coercivity

\section{Introduction}

Polycrystalline ferrites are of great interest as a result of their scientific characteristics and of potential applications in various electromagnetic devices. Lithium ferrite and substituted lithium ferrites are materials with attractive materials with excellent properties. Their low cost, high saturation magnetization, high Curie temperature, and hysteresis loop properties make them excellent candidates for high-density recording media, absorbers, and microwave devices $[1,2]$. It was previously known that polycrystalline Li-Zn ferrite has a relatively large Snoek's product value and that it is a potential candidate for application in high-frequency devices. Furthermore, it was found that a solid solution formation with $\mathrm{Cu}$ ferrite promoted low temperature densification and that the partial substitution of Fe by $\mathrm{Mn}$ enhanced the electrical resistivity. Recently, a multi-layer chip inductor was fabricated using a polycrystalline $\mathrm{Li}-\mathrm{Zn}-\mathrm{Cu}-\mathrm{Mn}$ ferrite via the

CThe Korean Magnetics Society. All rights reserved.

*Corresponding author: Tel: +82-43-840-3623

Fax: +82-43-851-4169, e-mail: kpchae@kku.ac.kr green-sheet technique, and its complex impedance spectrum was evaluated with the help of a numerical calculation $[3,4]$. In a previous study, we showed that Al-substituted Li-Co-Ti ferrites are well crystallized with a spinel structure and showed interesting crystallographic and magnetic properties of such materials [5]. In this study, we have synthesized $\mathrm{Li}_{0.5} \mathrm{Zn}_{0.2} \mathrm{Cu}_{0.4} \mathrm{Mn}_{\mathrm{x}} \mathrm{Fe}_{2.1-\mathrm{x}} \mathrm{O}_{4}(0.0 \leq \mathrm{x} \leq 0.8)$ ferrites and have studied them by X-ray diffractometry (XRD), field emission scanning electron microscopy (FESEM), Mössbauer spectroscopy, and vibrating sample magnetometry (VSM) to understand the influence of the $\mathrm{Mn}$ substitution in the scope of a spinel structure and ferrimagnetic property.

\section{Experiment}

Samples of the manganese substitution lithium zinc copper ferrite powders, $\mathrm{Li}_{0.5} \mathrm{Zn}_{0.2} \mathrm{Cu}_{0.4} \mathrm{Mn}_{\mathrm{x}} \mathrm{Fe}_{2.1-\mathrm{x}} \mathrm{O}_{4}$, with a manganese concentration ranging from $\mathrm{x}=0.0$ to 0.8 with a step of 0.2 , were prepared using a conventional ceramic method. The $\mathrm{Li}_{2} \mathrm{O}, \mathrm{ZnO}, \mathrm{CuO}, \mathrm{Fe}_{2} \mathrm{O}_{3}$, and $\mathrm{Mn}_{2} \mathrm{O}_{3}$ powders were all $99.99 \%$ pure. After the powders had been dried at $120^{\circ} \mathrm{C}$ for $2 \mathrm{~h}$, the mixtures were milled for $1 \mathrm{~h}$ and 
then were pressed into pellet form using a hydraulic press at $8 \mathrm{ton} / \mathrm{cm}^{2}$. These pellets were sintered in a furnace and were annealed at $900^{\circ} \mathrm{C}$ for $6 \mathrm{~h}$. The treatment was then followed by a second milling and the materials were pressed into pellet form once again, with an annealing process at $1,100^{\circ} \mathrm{C}$ for $6 \mathrm{~h}$. These samples were then used in powder form for the X-ray analysis and the Mössbauer experiment.

The X-ray diffraction (Philips X'pert-MPD) patterns of the powder samples were obtained at room temperature with $\operatorname{Cuk} \alpha(\lambda=1.5418 \AA)$ radiation, and the surface microstructures were observed using FESEM (Hitachi 3000) at room temperature. The Mössbauer spectra were obtained with a conventional electromechanical-type Mössbauer spectrometer (FAST Com. Tec.) with a ${ }^{57} \mathrm{Co}$ source $(10 \mathrm{mCi})$ in a rhodium matrix. Finally, the measurements of the magnetic properties were carried out using a VSM (Lake Shore 7404).

\section{Results and Discussion}

$\mathrm{X}$-ray diffraction patterns of the manganese substitution lithium-zinc-copper ferrites, $\mathrm{Li}_{0.5} \mathrm{Zn}_{0.2} \mathrm{Cu}_{0.4} \mathrm{Mn}_{\mathrm{x}} \mathrm{Fe}_{2.1-\mathrm{x}} \mathrm{O}_{4}$ $(0.0 \leq \mathrm{x} \leq 0.8)$, are shown in Fig. 1. The X-ray diffraction measurements show that all of the peaks of these samples are consistent with those of a typical spinel structure with an $F d 3 m$ space group. Furthermore, these measurements confirm the absence of any secondary phase. The value of

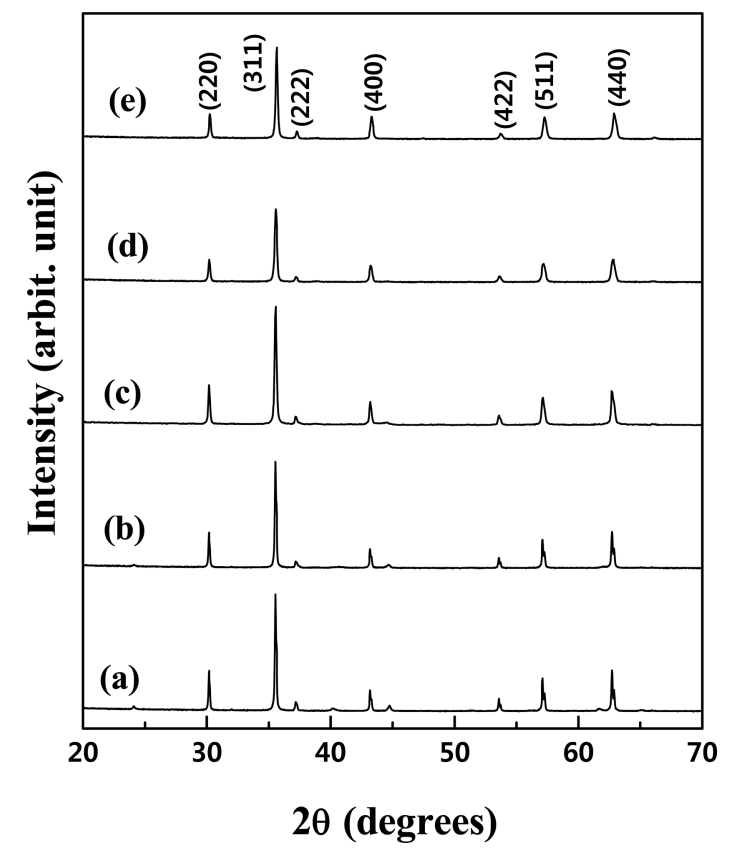

Fig. 1. X-ray diffraction patterns of $\mathrm{Li}_{0.5} \mathrm{Zn}_{0.2} \mathrm{Cu}_{0.4} \mathrm{Mn}_{\mathrm{x}} \mathrm{Fe}_{2.1 \text { - }}$ ${ }_{\mathrm{x}} \mathrm{O}_{4}$ ferrites: (a) $\mathrm{x}=0.0$, (b) $\mathrm{x}=0.2$, (c) $\mathrm{x}=0.4$, (d) $\mathrm{x}=0.6$ and (e) $\mathrm{x}=0.8$.

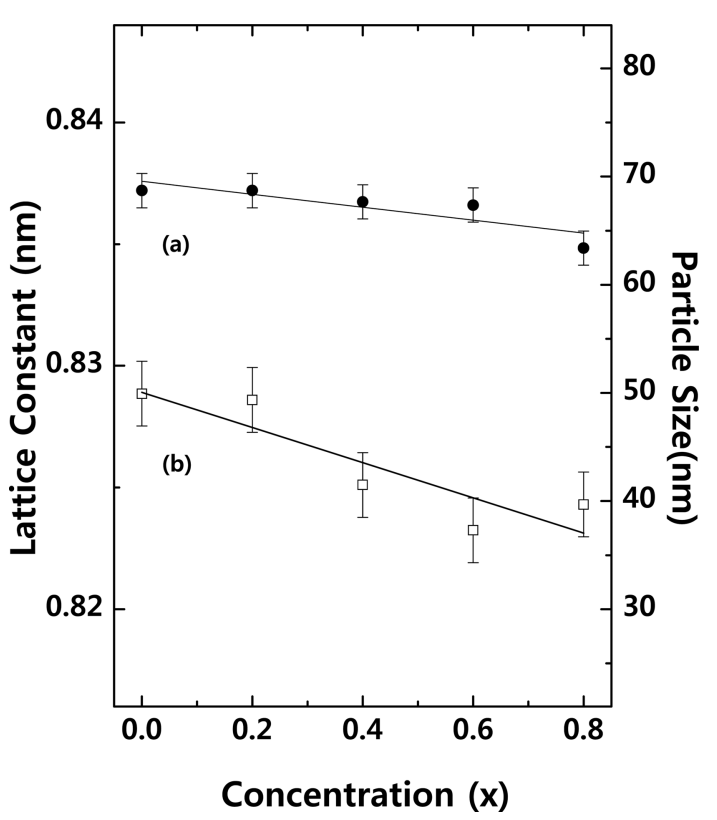

Fig. 2. Variation of lattice constants and particle sizes in $\mathrm{Li}_{0.5} \mathrm{Zn}_{0.2} \mathrm{Cu}_{0.4} \mathrm{Mn}_{\mathrm{x}} \mathrm{Fe}_{2.1-\mathrm{x}} \mathrm{O}_{4}$ ferrites.

the lattice constant $a$, as calculated from the observed $d$ values, linearly decreased within an acceptable range of experimental error of $0.837 \mathrm{~nm}$ for $\mathrm{x}=0.0$ and $0.835 \mathrm{~nm}$ for $\mathrm{x}=0.8$, as shown in Fig. 2. This can be explained by Vegard's law [6] which explains the linear variation in a lattice constant with an ionic radii of the doped and the replacing ion. In this study we replaced $\mathrm{Fe}^{3+}$ ions with $\mathrm{Mn}^{3+}$ ions since the ionic radius of $\mathrm{Mn}^{3+}$ ions are of 0.66 $\AA$, smaller than the radius of $\mathrm{Fe}^{3+}$ ions at $0.67 \AA$. Thus the substitution of $\mathrm{Fe}^{3+}$ ions with $\mathrm{Mn}^{3+}$ results in a decrease in the lattice constant of the ferrite.

The size of the particles was determined from the diffraction peak broadening of the Scherrer equation [7], $t$ $\left.=(0.9 \lambda) / B \cos \theta_{B}\right)$, where $\lambda$ represents the X-ray wavelength, $B$ is the half width of the (311) peak, and $\theta_{B}$ is the angle of the (311) peak. The size of the particles of $\mathrm{Li}_{0.5} \mathrm{Zn}_{0.2} \mathrm{Cu}_{0.4} \mathrm{Mn}_{\mathrm{x}} \mathrm{Fe}_{2.1-\mathrm{x}} \mathrm{O}_{4}$ ferrite powders also somewhat decreases as $49.9 \mathrm{~nm}(\mathrm{x}=0.0), 49.4 \mathrm{~nm}(\mathrm{x}=0.2), 41.5(\mathrm{x}$ $=0.4), 37.3(\mathrm{x}=0.6)$, and $39.7 \mathrm{~nm}(\mathrm{x}=0.8)$, as shown in Fig. 2. Similar results were obtained for Li-Co-Ti ferrites [8].

Fig. 3 shows the FESEM images of $\mathrm{Li}_{0.5} \mathrm{Zn}_{0.2} \mathrm{Cu}_{0.4}$ $\mathrm{Mn}_{\mathrm{x}} \mathrm{Fe}_{2.1-\mathrm{x}} \mathrm{O}_{4}$ ferrites. These photomicrographs were taken under a magnification of 5,000x at the same scale for all samples. It can be seen that the average grain size and/or morphology of the $\mathrm{Li}_{0.5} \mathrm{Zn}_{0.2} \mathrm{Cu}_{0.4} \mathrm{Mn}_{\mathrm{x}} \mathrm{Fe}_{2.1-\mathrm{x}} \mathrm{O}_{4}$ ferrite depend on the manganese substitution. As the manganese substitution increases, some smaller grains were found to be embedded among the bigger grains, and these increased 


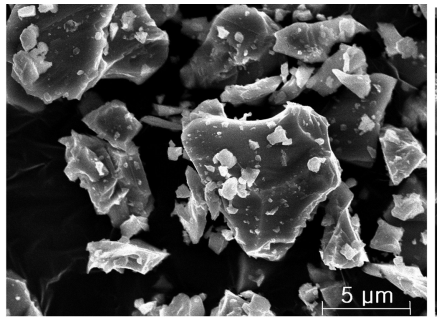

(a)

(c)

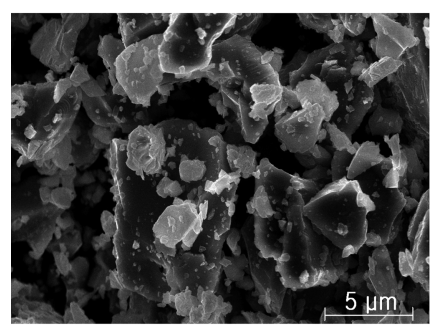

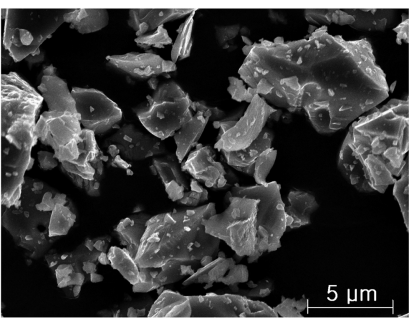

(b)

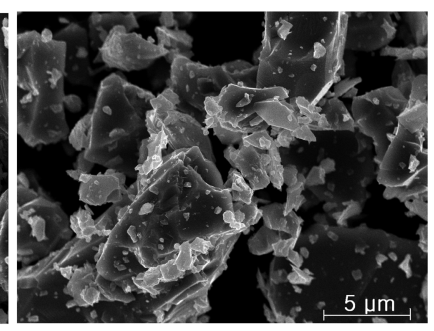

(d)
Fig. 3. FESEM images of $\mathrm{Li}_{0.5} \mathrm{Zn}_{0.2} \mathrm{Cu}_{0.4} \mathrm{Mn}_{\mathrm{x}} \mathrm{Fe}_{2.1-\mathrm{x}} \mathrm{O}_{4}$ ferrites: (a) $\mathrm{x}=0.0$, (b) $\mathrm{x}=0.2$, (c) $\mathrm{x}=0.4$ and (d) $\mathrm{x}=0.8$.

in number. This means that the manganese substitution in $\mathrm{Li}-\mathrm{Zn}-\mathrm{Cu}$ ferrite disturbed grain growth, and similar results were obtained for aluminum-substituted Li-Co-Ti ferrites [5]. S. A. Manzen et al. [10] showed that the lattice parameter and average grain diameter increase with increasing $\mathrm{Mn}$ ion substitution in $\mathrm{Li}_{0.5-0.5 \mathrm{x}} \mathrm{Mn}_{\mathrm{x}} \mathrm{Fe}_{2.5-0.5 \mathrm{x}} \mathrm{O}_{4}$. But in our $\mathrm{Li}_{0.5} \mathrm{Zn}_{0.2} \mathrm{Cu}_{0.4} \mathrm{Mn}_{\mathrm{x}} \mathrm{Fe}_{2.1-\mathrm{x}} \mathrm{O}_{4}$ sample, we found that the lattice parameter and the average grain diameter decreased with increasing $\mathrm{Mn}$ ion substitution.

The X-ray results show that the low substitution of manganese leads to no other phase in the spinel structure, and the Mössbauer spectrum provides a means of studying the microstructural properties of the substituted

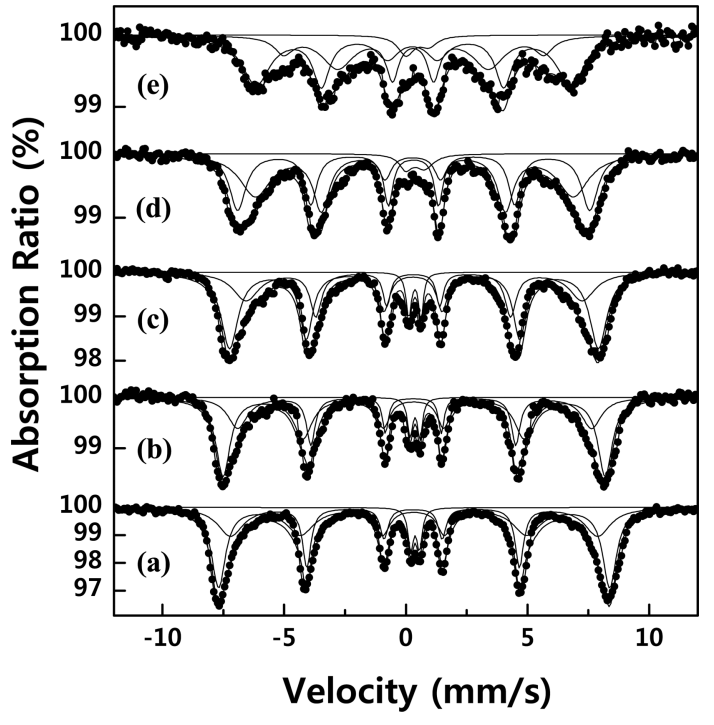

Fig. 4. Mössbauer spectra at room temperature of $\mathrm{Li}_{0.5} \mathrm{Zn}_{0.2^{-}}$ $\mathrm{Cu}_{0.4} \mathrm{Mn}_{\mathrm{x}} \mathrm{Fe}_{2.1-\mathrm{x}} \mathrm{O}_{4}$ ferrites: (a) $\mathrm{x}=0.0$, (b) $\mathrm{x}=0.2$, (c) $\mathrm{x}=0.4$, (d) $\mathrm{x}=0.6$ and (e) $\mathrm{x}=0.8$.

systems in a way that X-ray diffraction cannot. Therefore, is is not of practical value in the study of substituted ferrites.

The Mössbauer absorption spectra measured at room temperature for $\mathrm{Li}_{0.5} \mathrm{Zn}_{0.2} \mathrm{Cu}_{0.4} \mathrm{Mn}_{\mathrm{x}} \mathrm{Fe}_{2.1-\mathrm{x}} \mathrm{O}_{4}$ are shown in Fig. 4, and all the spectra of the samples are fitted with two sextet subspectra assigned to $\mathrm{Fe}^{3+}$ at the tetrahedral A-site and octahedral B-sites of a typical spinel crystal structure, and of one doublet, as shown in Fig. 4 and Table 1. The isomer shift (IS) values at room temperature are $0.30-0.43 \mathrm{~mm} / \mathrm{s}$ relative to the $\mathrm{Fe}$ metal and are consistent with a high-spin $\mathrm{Fe}^{3+}$ charge state. The quadrupole

Table 1. Room-temperature Mössbauer parameters of $\mathrm{Li}_{0.5} \mathrm{Zn}_{0.2} \mathrm{Cu}_{0.4} \mathrm{Mn}_{\mathrm{x}} \mathrm{Fe}_{2.1-\mathrm{x}} \mathrm{O}_{4} . H_{h f}$ is the magnetic hyperfine field, $Q S$ is the quadrupole splitting, and $I S$ is the isomer shift relative to the metallic iron. Area ratio is the absorption ratios of the A- and B-sites in the Zeeman sextets, and the doublet in the Mössbauer spectra.

\begin{tabular}{|c|c|c|c|c|c|c|c|c|c|}
\hline & \multirow{3}{*}{ spectrum } & \multicolumn{2}{|c|}{$H_{h f}(\mathrm{kOe})$} & \multicolumn{2}{|c|}{$Q S(\mathrm{~mm} / \mathrm{s})$} & \multicolumn{2}{|c|}{$I S(\mathrm{~mm} / \mathrm{s})$} & \multicolumn{2}{|c|}{ area ratio $(\%)$} \\
\hline & & A-site & B-site & A-site & B-site & A-site & B-site & A-site & B-site \\
\hline & & & & & & & & & \\
\hline \multirow[t]{2}{*}{0.0} & sextet & 468.0 & 498.5 & 0.02 & 0.01 & 0.31 & 0.32 & 38.0 & 56.6 \\
\hline & doublet & & & & & & & & \\
\hline \multirow[t]{2}{*}{0.2} & sextet & 452.6 & 487.8 & 0.02 & 0.01 & 0.33 & 0.32 & 35.6 & 59.2 \\
\hline & doublet & & & & & & & & \\
\hline \multirow[t]{2}{*}{0.4} & sextet & 428.5 & 470.0 & 0.01 & 0.01 & 0.32 & 0.31 & 33.7 & 61.2 \\
\hline & doublet & & & & & & & & \\
\hline \multirow{2}{*}{0.6} & sextet & 387.9 & 441.6 & 0.01 & 0.02 & 0.33 & 0.31 & 31.9 & 63.2 \\
\hline & doublet & & & & & & & & \\
\hline \multirow{2}{*}{0.8} & sextet & 330.0 & 400.6 & 0.01 & 0.01 & 0.31 & 0.30 & 36.2 & 56.0 \\
\hline & doublet & \multicolumn{2}{|c|}{ - } & \multicolumn{2}{|c|}{0.92} & \multicolumn{2}{|c|}{0.43} & \multicolumn{2}{|c|}{7.8} \\
\hline
\end{tabular}


splitting $(Q S)$ values for both the A- and B-sites of the Zeeman sextets are almost 0 , which is in accordance with the cubic crystal structure. The Fe ions that do not participate in the superexchange process in the spinel structure will show a doublet in the Mössbauer spectrum, with similar results appearing in the aluminum-substituted and the vanadium-substituted Li-Co-Ti ferrite [5, 9].

As shown in Table 1, the isomer shift and the quadruple splitting values are nearly constant in the Zeeman sextets with substituted manganese contents. However, the Mössbauer parameters show a decrease in $H_{h f}$ from the substituted manganese content increase. This indicates that the substitution of magnetic manganese ions has an influence on the superexchange interaction in the A- and B-sites of the spinel structure.

Using the site preference and Mössbauer spectra area ratio, the cation distribution can be determined. The cation distribution depends on many factors, including the temperature, pressure, and composition, as well as the compound preparation method. From Fig. 4 we can see that the area ratio of the A- and B-sites in the Zeeman sextets shows small decreases with an increasing concentration of substituted manganese. Using lithium and copper ions located in the B-site and the zinc ions that enter into the A-site $[11,12]$, we can state that the cation

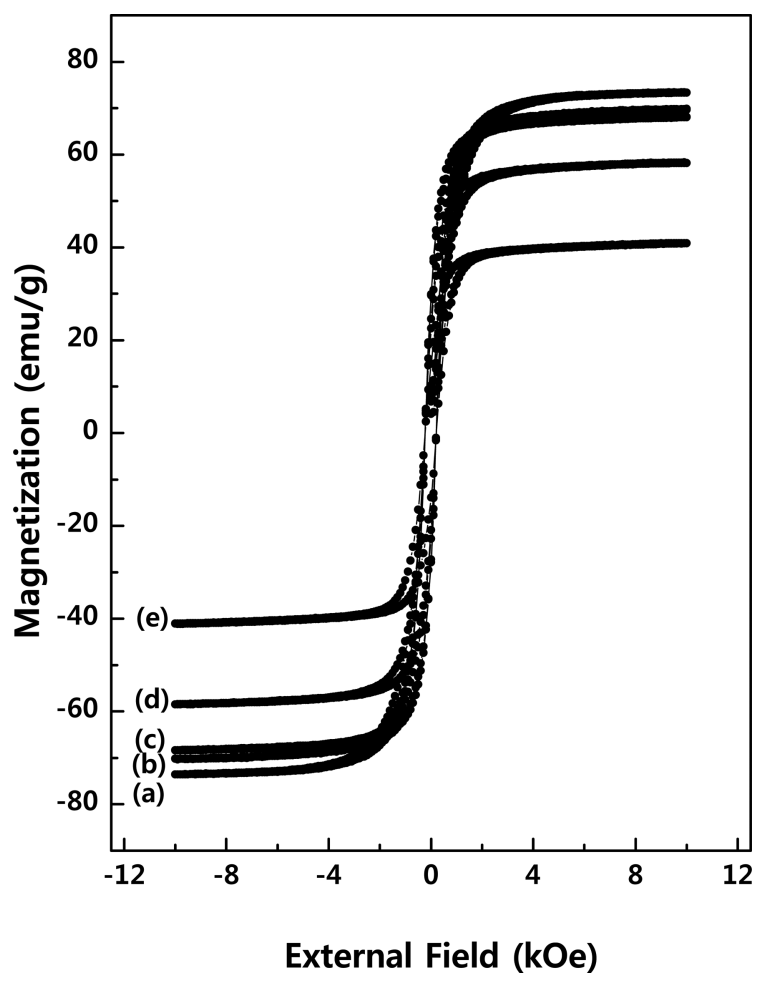

Fig. 5. Hysteresis curves of $\mathrm{Li}_{0.5} \mathrm{Zn}_{0.2} \mathrm{Cu}_{0.4} \mathrm{Mn}_{\mathrm{x}} \mathrm{Fe}_{2.1-\mathrm{x}} \mathrm{O}_{4}$ ferrites: (a) $\mathrm{x}=0.0$, (b) $\mathrm{x}=0.2$, (c) $\mathrm{x}=0.4$, (d) $\mathrm{x}=0.6$ and (e) $\mathrm{x}$ $=0.8$. distribution from the non-relaxation Mössbauer spectra $(0.0 \leq \mathrm{x} \leq 0.6)$ area ratio is represented by $\left(\mathrm{Zn}_{0.2} \mathrm{Mn}_{0.5 \mathrm{x}} \mathrm{Fe}_{0.8-0.5 \mathrm{x}}\right)$ $\left[\mathrm{Li}_{0.5} \mathrm{Cu}_{0.4} \mathrm{Mn}_{0.5 \mathrm{x}} \mathrm{Fe}_{1.2-0.5 \mathrm{x}}\right] \mathrm{O}_{4}$.

The Fe ions that do not participate in the superexchange process in the spinel structure show a doublet in the Mössbauer spectrum. About $94.6 \%$ (for $\mathrm{x}=0.0$ ) and about $95.1 \%$ (for $\mathrm{x}=0.6$ ) of the $\mathrm{Fe}$ ions participate in the superexchange process of this spinel structure, and about $5.4 \%$ (for $\mathrm{x}=0.0$ ) and $4.9 \%$ (for $\mathrm{x}=0.6$ ) do not participate in the superexchange process. That is, $\mathrm{Fe}^{3+}$ ions that escaped and/or overflowed from the B-site do not participate in the A-O-B superexchange interaction, so they show quadruple splitting in the Mössbauer spectra.

The magnetic properties of the samples were determined at room temperature using a vibrating sample magnetometer. In Fig. 5, we can see the hysteresis curve, and in Fig. 6 the changes in the saturation magnetization $\left(M_{S}\right)$ and the coercivity $\left(H_{C}\right)$ with a maximal field of $10 \mathrm{kOe}$. The values of the samples' coercivity range from 220 Oe to $230 \mathrm{Oe}$, indicating that samples are soft magnetic. As manganese concentration increases, the saturation magnetization decreases with coercivity remaining nearly constant. The maximum saturation magnetization is 73.35 $\mathrm{emu} / \mathrm{g}$ at $\mathrm{x}=0.0$, and the coercivity is $220-230$ Oe. The variation of the saturation magnetization can be explained with Neel's two sub-lattice models [13]. The magnetic moment in the inverse ferrites is mainly due to the

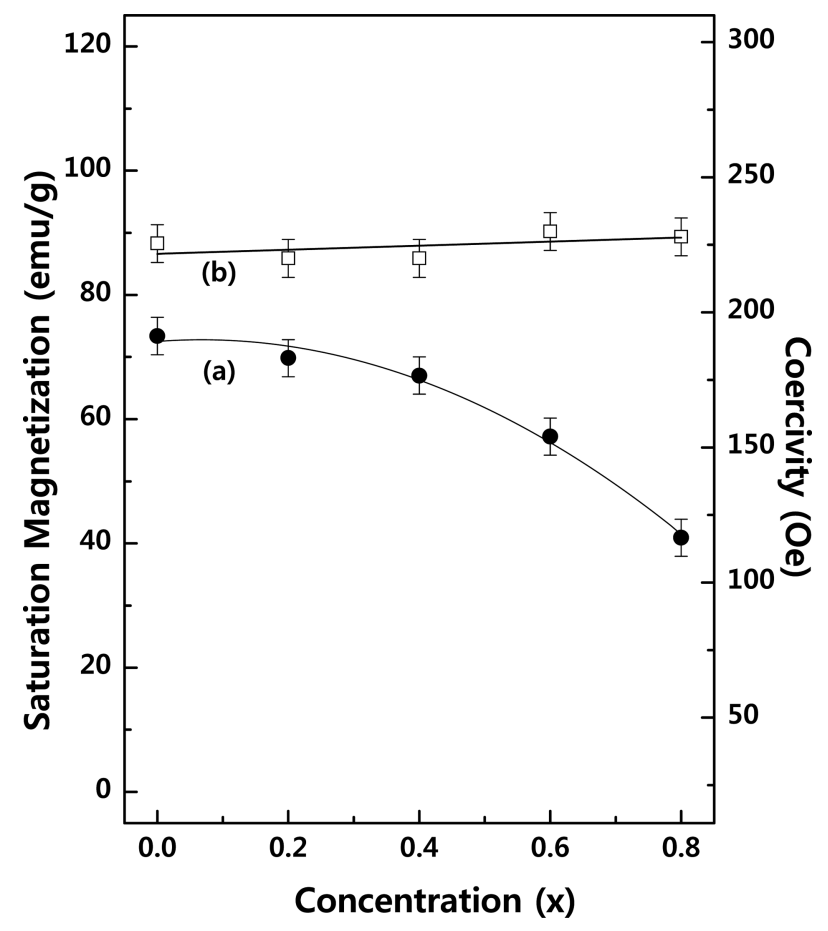

Fig. 6. (a) Saturation magnetization and (b) coercivity of $\mathrm{Li}_{0.5} \mathrm{Zn}_{0.2} \mathrm{Cu}_{0.4} \mathrm{Mn}_{\mathrm{x}} \mathrm{Fe}_{2.1-\mathrm{x}} \mathrm{O}_{4}$ ferrites. 
resultant magnetic moments of the A- and B-sites. The A$B$ superexchange interaction is the predominant interaction, but not in the sublattice A-A and B-B interactions. Hence the net magnetic moment is given as $\mu=\left(\mu_{B}-\mu_{A}\right)$ because we substituted a magnetic ion $\mathrm{Fe}^{3+}\left(5 \mu_{\mathrm{B}}\right)$ with $\mathrm{Mn}^{3+}\left(4 \mu_{\mathrm{B}}\right)$ in the A- and B-site, resulting in a decrease in the net magnetic moment of the manganese-substituted $\mathrm{Li}-\mathrm{Zn}-\mathrm{Cu}$ ferrite. It is known that the coercivity generally decreases with a decrease in particle size as a result of the lower anisotropy barriers [14]. In our samples, the coercivity is nearly constant, as shown in Fig. 6, which can be also related to the particle size variation as shown in Fig. 3. In our case, coercivity variation is due to a combination of the particle size variation and the mentioned effect of the reduced interaction after an increase of Mn content.

\section{Conclusion}

The manganese-substituted lithium zinc copper ferrites, $\mathrm{Li}_{0.5} \mathrm{Zn}_{0.2} \mathrm{Cu}_{0.4} \mathrm{Mn}_{\mathrm{x}} \mathrm{Fe}_{2.1-\mathrm{x}} \mathrm{O}_{4}(0.0 \leq \mathrm{x} \leq 0.8)$, showed $\mathrm{a}$ single phase with a spinel structure, and their lattice constants linearly decreased and the particle size somewhat decreased as the substituted Mn content increased. All the Mössbauer spectra could be fitted with two Zeeman sextets, which are the typical spinel ferrite spectra of $\mathrm{Fe}^{3+}$ with A- and B-sites, and one doublet. Using the area ratio of the Mössbauer spectra, the cation distribution could be determined as $\left(\mathrm{Zn}_{0.2} \mathrm{Mn}_{0.5 \mathrm{x}} \mathrm{Fe}_{0.8-0.5 \mathrm{x}}\right)\left[\mathrm{Li}_{0.5} \mathrm{Cu}_{0.4} \mathrm{Mn}_{0.5 \mathrm{x}} \mathrm{Fe}_{1.2-0.5 \mathrm{x}}\right]$. The magnetic behavior of the samples showed that an increase in the manganese contents led to a decrease in the saturation magnetization, whereas coercivity was nearly constant. The variation of the saturation magnetization could be explained with Neel's two sub-lattice models.

\section{Acknowledgements}

This paper was written as part of Konkuk University's research support program for its faculty on sabbatical leave in 2014.

\section{References}

[1] X. Cao, K. Sun, C. Sun, and L. Leng, J. Magn. Magn. Mater 321, 2896 (2009).

[2] S. A. Jadhav, J. Magn. Magn. Mater. 224, 167 (2001).

[3] T. Nakamura, T. Miyamoto, and Y. Yamada, J. Magn. Magn. Mater. 256, 340 (2003).

[4] T. Nakamura, M. Naoe, and Y. Yamada, J. Magn. Magn. Mater. 305, 120 (2006).

[5] J. K. Kang, W. H. Kwon, J. G. Lee, K. P. Chae, and Y. B. Lee, J. Korean Phys. Soc. 59, 85 (2011).

[6] C. G. Whinfrey, D. W. Eckort, and A. Tauber, J. Am. Chem. Soc. 82, 2695 (1960).

[7] B. D. Cullity, Elements of X-Ray Diffraction, AdditionWesley Co. Readings, MA (1978) p. 102.

[8] K. P. Chae, W. H. Kwon, and J. G. Lee, J. Magn. Magn. Mater. 324, 2701 (2012).

[9] W. H. Kwon, J. G. Lee, S. W. Lee, and K. P. Chae, J. Korean Phys. Soc. 56, 1838 (2010).

[10] S. A. Mazen and N. I. Abu-Elsaad, J. Magn. Magn. Mater. 324, 3366 (2012).

[11] S. A. Jadhav, Mater. Chem. Phys. 65, 120 (2000).

[12] M. Maisnam, S. Phanjoubam, H. N. K. Sarma, C. Prakash, L. R. Devi, and O. P. Thakur, Materials Lett. 58, 2412 (2004).

[13] L. Neel, Ann. Phys. 3, 137 (1948).

[14] C. P. Bean and J. D. Livingston, J. Appl. Phys. 30, 120S (1959). 